\title{
Editorial: Special Issue on "Wireless Innovation for Inter-dynamic Technology (WIDTH)" (Selected Topics from the Strategic Workshop, May 15-17, 2009, Rebild, Denmark)
}

\author{
Marina Ruggieri • Ole Brun Madsen
}

Published online: 18 March 2010

(C) Springer Science+Business Media, LLC. 2010

The 11th Edition of the closed-door Strategic Workshop was held in Rebild, Denmark, from 15th to 17th of May, 2009. The focus during the active two-day discussions of the participating major stakeholders, was on wireless innovation for inter-dynamic technology.

Inter-dynamic technology is the next step on the road towards converged next generation communication infrastructures. The main characteristic of that scenario is heterogeneity, caused by large-scaled networks, even larger number of nodes, closely or remotely located and the need for making these cooperate in order to deliver services seamlessly across multiple domains. Resources in such an environment are needed for ensuring among others also reliability of the communicated information. The objective of the event was to promote the convergence of interest with industrial commitment and leadership in determining strategic research agendas.

This Special Issue features ten selected papers on the above topic that focus on various innovative proposals that can enable inter-dynamic technology towards a converged Information and Communication Technology (ICT) infrastructure.

The first paper, "Future Scenarios-What are the Future Services and Applications?" by Christian Kloch, Jens Enevold Kristensen and Bent Bilstrup, presents thoughts on a new ICT paradigm within user-machine interaction hwere seamless interoperability between different technologies is crucial for enabling novel applications and services.

The second paper, "A Novel Approach for Physical Layer Cryptography in Wireless Networks," by Lorenzo Mucchi, Luca Simone Ronga and Enrico del Re, focuses on a very important issue of how to secure the wireless communication exchange. The contribution describes a novel approach to physical layer security.

\footnotetext{
M. Ruggieri $(\varangle)$

Department of Electronics Eng., CTIF-Italy, University Roma Tor Vergata,

Via Politecnico 1, 00133 Rome, Italy

e-mail: ruggieri@uniroma2.it

O. B. Madsen

CTIF, Aalborg University, Inst. 8-Kommunikationsteknologi,

Niels Jernes Vej 12, Room a5-213, 9220 Aalborg, Denmark

e-mail: obm@es.aau.dk
} 
The third paper, "Cooperative Communications in Future Home Networks" by Zülkülf Genc, Umar H. Rizvi, Ertan Onur and Ignas Niemegeers, proposes a strategy for improving end-to-end performance in wireless cellular and ad hoc networks and investigates the potential gains of cooperative communication in future home networks. The main idea is to use very high frequency bands $(60 \mathrm{GHz})$ for satisfying the demand for very high data rates in the home.

The fourth paper, "Wireless Innovations as Enablers for Complex and Dynamic Large Artificial Systems" by Spyridon Vassilaras and Gregory S. Yovanof discusses the complexity and criticality of large-scaled ICT systems for a variety of applications (e.g., healthcare, smart urban environments, homeland security, etc). Theoretical and practical issues as well as research directions are presented, including theoretical link and network capacity limits, cognitive radio and cognitive networking, complex system design, and so forth.

The fifth paper, "A Living Laboratory Exploring Mobile Support for Everyday Life with Diabetes," by Kim Bjerge and Jens Enevold Kristensen presents the set up of a Living Laboratory in a city in the North of Denmark, and exploring mobile support for everyday life with diabetes. The living-lab method combined with a user-driven approach is an example of how designers can carry out and gain from working with people as co-creators in design projects.

The sixth paper, "Advancing Radio Testing Methodology via Formal Notations," by J. Poncela, J. T. Entrambasaguas, and Mari Carmen Aguayo-Torres, proposes a verification methodology for the correct operation of complex ICT equipment in public environments. It is shown how the design process can be merged for both protocol and radio test systems, and what are the requirements for instrumentation and standardisation of the control interfaces.

The seventh paper, "Improved ICT Infrastructure," by Ole Brun Madsen discusses the challenges in design and management of the next generation ICT infrastructure where communication between distributed nodes and entities is a key characteristic. A reference architecture is also proposed.

The eighth paper, "Users, Economics, Technology: Unavoidable Interdynamics" by Rui L. Aguiar, Ertan Onur, Knud Erik Skovby, Mari-Carmen Aguayo-Torres, and Thomas Toftegaard Nielsen presents thoughts and directions on how ICT technology is evolving and what challenges lie ahead for satisfying the design, user and design requirements.

The ninth paper, "Strategies and Challenges for Interconnecting Wireless Mesh and Wireless Sensor Networks" by Stefan Bouckaert, Eli de Poorter, Benôit Latré, Jeroen Hoebeke, Ingrid Moerman, and Piet Demeester, proposes solutions for future heterogeneous network environments. Convergence of layers and the development of adaptive network protocols is presented as a promising approach to enable communication in heterogeneous environments.

The tenth paper, "Next Generation Wireless Infrastructure" by Thomas Skjødeberg Toftegaard proposes new ways of using network intelligence by means of more network and device cooperation. The goal is to build an ICT platform that can support major application areas of society and economy. 


\section{Author Biographies}

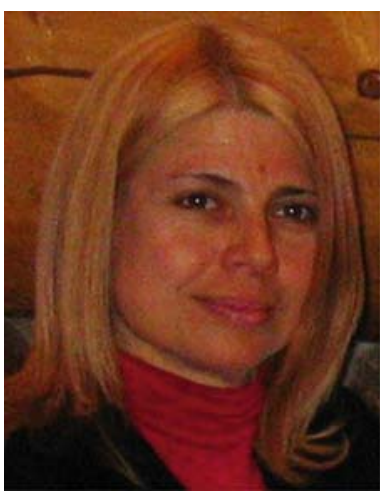

Marina Ruggieri is Full Professor in Telecommunications at the University of Roma Tor Vergata. Since 2003 she directs there a M.Sc. in Advanced Satellite Communications and Navigation Systems. Her research focuses on space communications and navigation systems, integrated systems, mobile and multimedia networks, ICT for biotechnology and energy. Since January 2010 she is President of the IEEE Aerospace and Electronic Systems (AES) Society. Since December 2006 she is Vice President of the AFCEA Rome Chapter. In 2004-2006 she was in the Technical-Scientific Committee of the Italian Space Agency (ASI). In 2007-2008 she has been Vice-President of the ASI Technical-Scientific Committee. Since December 2007 she belongs to the Italian Superior Council of Telecommunications as Expert. She is Director of CTIF_Italy, the Italian branch of the Center for Teleinfrastruktur (CTIF) in Aalborg (Danimarca), opened on September 28, 2006 at Tor Vergata. She is Editor of the IEEE Transactions on AES. She received the 1990 Piero Fanti International Prize, the 2009 Pisa Woman award and the nomination for the 1996 Harry M.Mimmo Award and the 2002 Cristoforo Colombo Award. She is author of about 250 papers, on international journals/transactions and proceedings of international conferences, book chapters and books (8).

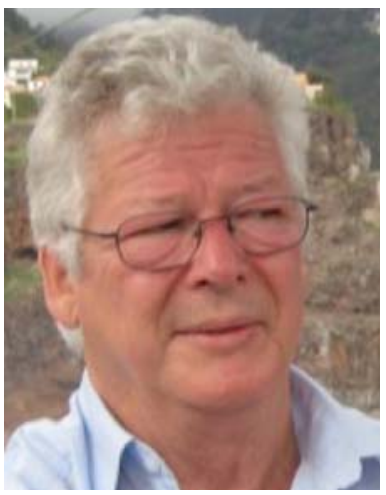

Ole Brun Madsen was born in 1942 in Denmark and received his M.Sc. in Mathematics \& Computer Science from the University of Copenhagen. (1962-1972) researcher and from 1968 head of the Computer Science Laboratory at The Royal Danish Academy of Fine Arts in Copenhagen. (1972-1981) head of the Development Department, RECAU, the Regional Computing Centre at Århus University. (1981-1996) Head of the Data Network Section and Head of the Network Infrastructure Strategy section at Jutland Telephone. (1996-1999) Manager for Infrastructure Network Technology and Strategy at TDC, Tele Denmark. (1999-) Professor in Distributed real-time Systems and from 2004 as Head of CNP, Center for Network Planning and Co-director for CTIF, Center for TeleInFrastructur at Aalborg University. He has been project leader for a number of national and international R\&D projects and acted in high level advisory tasks within the European Commission on the R\&D framework programmes in DGXIII and with United Nations UNDP activities. Present research is focused on Infrastructure Architecture and Modelling Tools for Network Analysis and Design. 